\title{
Vida de Carmen Caamaño Díaz: Una voz del exilio interior
}

\author{
Mercedes Montero Caldera *
}

\section{RESUMEN ABSTRACT}

Este antículo recoge el testimonio oral de Carmen Caamaño Diaz (Madrid, 1911), quien a través de su memoria hace un recorrido autobiográfico en el que manifiesta la evolución de un compromiso ideológico que la convertiría en sujeto activo del exilio interior durante el régimen franquista. En su etapa universitaria, Carmen fue miembro de la junta Directiva de la FUE (Federación Española de Enseñantes) y Secretaria General de la UFEH (Unión Federal de estudiantes Hispanoamericanos) desde 1930 a 1931. Licenciada en Filosofia y Letras, trabajó en el Centro de estudios historicos formando parte del equipo

de investigación que dirigía Claudio Sánchez Albornoz. En 1937 ingresó en el P.C.E, desempeñando, entre otros cargos, el de Gobernadora Civil de Cuenca desde
This article includes the oral statement of Carmen Caamaño Diaz (Madrid, 1911), who covers her autobiography through her memory, expressing an ideogical compromise evolution through which she would become an active subjet of the inner exile throughout Franco's regime. While being a student at university, she was a member of the Board of Directors of the UFEH (Spanish Teachers Union) and General Secretary of the UFEH (Latin American Student Union) from 1930 to 1931. With a degree in Atrs, she worked at the Centre of Historic Studies taking part within the investigation team conducted by Claudio Sánchez Albornoz. In 1937 she joined the Spanish Communist Party, performing the charge, amongst others, of Civil Governor of Cuenca from January to March 1939. At the end of the war, she was arrested

* Departamento de Historia Contemporánea. (UNED). 
enero a marzo de 1939.

Al finalizar la guerra, fue detenida y condenada a prisión. En 1941 trató de reorganizar en la clandestinidad el partido comunista en la provincia de Alicante, siendo nuevamente detenida y condenada a muerte, pena que le sería conmutada por la de cárcel. Depurada profesionalmente y bajo control policial durante varios años, su actividad de oposición al régimen de

Franco la llevaria a cabo desde la Asociación de Mujeres Universitarias.

PALABRAS CLAVE

Testimonio oral. Memoria historica. Segunda Republica. Guerra Civil. Partido Comunista de España. Régimen de Franco. Exilio interior. and sentenced to imprisoment. In 1941 she tried to reorganise in secret the Communist Party in the province of Alicante. She was again arrested and sentenced to the death penalty, penalty that would be commuted for imprisonment. Politically purged and under police control throughout several years, she would undertake her opposition activity to Franco's regime from the University Women Association.

\section{KEY WOROS}

Oral statement, Historic memory, Second Republic, Civil war, Spanish communist Party, Franco's Regime, inner exile.

\section{INFANCIA Y ADOLESCENCIA (1911-1925)}

Un amigo de Carmen, Arturo Soria y Espinosa, nieto del urbanista Arturo Soria y Mata, solia decir "La vida de la mayoría de los hombres trascurre entre el ilusionismo estéril y la obcecación en el error» '. Tratandose de ellos dos y de muchos hombres y mujeres de su generación, "la de la guerra", se puede afirmar que se sustrajeron de ambos peligros a pesar de que la dureza del tiempo que les tocó vivir amenazó con romper sus muchas iniciativas y reducir sus ganas de trasformar el mundo en mera obcecación.

Las palabras de Carmen dejan siempre entrever un compromiso humano e ideológico sostenidos a lo largo de una vida. Hay en su persona dos rasgos físicos que llaman la atención a quien la conoce por primera vez, una voz segura y convincente, y unos ojos azules brillantes y vivarachos que observan lo que les rodea con una curiosidad que sus noventa años recién cumplidos mantienen intacta.

Carmen vive desde hace más de cuarenta años en una casa del barrio madrileño de Chamberí, y si como se dice, la casa es una prolonga-

\footnotetext{
SORIA, A.: "Ortega: primer y último encuentro", Revista de Occidente, núm 26, (1983), p. 77.
} 
ción de la personalidad de quien la habita, en el caso de Carmen, esta afirmación queda patente solo entrar en ella. Libros y cuadros aparecen por todas partes en un orden perfecto. Algunos fueron pintados por Ricardo Fuente ${ }^{2}$ " "Señor de Caamaño", como él solia decir humorísticamente, para romper la tradicional norma de presentación social: "esta es la señora de fulanito...». Ricardo fue dibujante en el diario El Sol cuando las caricaturas de Bagaría resumían, en unos pocos trazos brillantes y agudos, la complicada situación que en todos los órdenes atravesaban nuestro país y el mundo durante la primera treintena del siglo. Una de ellas cuelga en una de las paredes del recibidor.

En Carmen los acontecimientos que determinaron la historia de España, desde los años veinte hasta la década de los ochenta, no sólo han condicionado su propia trayectoria vital sino que ésta, por la implicación que decidió tener desde su juventud, aparece entrelazada a ellos tanto en el ámbito de lo privado, amistades, matrimonio, maternidad..., como en su proyección social, como veremos a través de las organizaciones culturales y politicas de las que formó parte activa y que constituían la vanguardia que luchaba por trasformar una sociedad atrasada, con unas estructuras decimonónicas, basadas en el privilegio y en una rigida estratificación de clases sociales, en una, en la que, como Carmen repetirá a lo largo de su testimonio, "todos accedieran según su capacidad personal, no social, ni económica a las mismas oportunidades". Su línea de pensamiento, y en coherencia con ella, su línea de actuación, han estado y están dentro del solidarismo social más que de la ortodoxia marxista, porque como ella afirma: "mi marxismo, es un marxismo de corazón, más que de pensamiento". Y ésta es la clave de toda una vida en la que "siempre hay que estar dispuesto a cambiar las cosas".

Como no podría ser de otro modo, el ambiente familiar y especialmente la figura paterna, querida y admirada, como resultado habitual en las mujeres de ideologia más progresista de esa generación, fue determinante en el arranquie de su trayectoria. La infancia y juventud de Carmen se va configurando dentro de la burguesía republicana de izquierda, cuyo objetivo, especialmente desde la dictadura de Primo de Rivera, el antecedente histórico más inmediato que en relación a Carmen interesa, era traer

\footnotetext{
Ricardo Fuente fue hijo de Ricardo Asensio, fundador junto a Francisco Ruano Caniedo de la Hemeroteca Municipal de Madrid en 1918, y primer director. Asimismo fue uno de los directores del diario El Pais, órgano de expresión del republicanismo radical de finales del xix y comienzos del xx. Sobre su actividad al frente del mismo Vid Álvarez JunCo, J., El Emperador del Paralelo. Lerroux y la demagogia populista. Madrid, 1998, pássim.
} 
a España una república democrática, laica y antioligárquica. Partiendo de la complejidad que caracterizaba a las clases medias de ese momento hay que decir que, si bien éstas se consideran culturalmente burguesas, no estaban vinculadas a la burguesía como clase económicamente hegemónica.

Por otra parte, es clave para comprender el testimonio de Carmen, en todo su sentido, dos grandes líneas que dividían la política: la que separaba a las organizaciones burguesas de las proletarias, y la que oponía derechas e izquierdas. Asi como la primera suponía esencialmente una contraposición social, aun con implicaciones sociales era basicamente una cuestión de afinidades ideológicas, no siendo excesivo afirmar, como evidencian las palabras y actitudes de Carmen y las de otras personas a las que ella aludirá expresamente, que la postura ante la Iglesia era la principal piedra de toque ${ }^{3}$.

Pero además, la izquierda republicana española, al igual que su homóloga europea, tenia que resolver una cuestión pendiente, que seria «el grito de guerra del siglo $x x "{ }^{4}$ : la cuestión social, frente a la cual hubo de proceder a un reajuste doctrinal a fin de conciliar su tradición liberal, que rechazaba cualquier conservadurismo, con los nuevos propósitos de reforma social. Dentro de esta evolución se manifestaba también una tendencia cada vez más significativa en ciertos sectores próximos a aquellos en los que Carmen iba construyendo su propia percepción de la realidad y de su cuota de responsabilidad ante ella hacia ideales revolucionarios. Tales planteamientos cobraron cada vez más rigor y pragmatismo a medida que, una vez proclamada la segunda República y pasado el tiempo de la esperanza, se fue poniendo de manifiesto la enorme dificultad de lograr por medios parlamentarios la reforma de la sociedad y del estado español. Acontecimientos como el auge de la derecha en las elecciones del 33, la revolución de Asturias del 34 y el auge del fascismo fuera y dentro de nuestro país, hicieron necesarios unos planteamientos cada vez más agresivos hacia los privilegios de unas minorias que continuaban intactos frente a la cada vez más deteriorada situación de las clases populares y también de amplios sectores de las clases medias.

Esta evolución ideológica se sigue paso a paso a través de los recuerdos que va desgranando la memoria de Carmen, en los que la influencia de un padre "liberal de izquierdas" aparece como esencial y diferenciada, en

Vid. Avilés Farré, J., La izquierda burguesa en la /l República. Madrid, 1985, p. 35

Aviles FARRE, J., op cit., p. 316. 
este sentido, del talante más conservador de la madre, en la formación tanto de sus hijos como de sus dos hijas Así recuerda que durante la infancia, su padre, funcionario del Ministerio de Fomento, "estaba muy pendiente de la situación de la calle», aludiendo a los disturbios que tuvieron lugar en varias ciudades españolas, entre ellas Madrid, durante el mes de noviembre de 1920, conocidos popularmente como «las huelgas del pan " 5 , y continua diciendo que: "en casa eso siempre se ha vivido no a favor de los que golpeaban, sino a favor de los golpeados". Esta preocupación paterna por la situación social y política, sería compartida tanto por sus dos hijos como por sus dos hijas ya que en este aspecto tan esencial en la educación de las personas y tan determinante de su actitud ante la vida de cara al futuro, recuerda que : "mi padre era poco prototipo de la época». "A todos nos dijo que cada uno era dueño de su propia persona". Son palabras que irán configurando toda una línea de conducta y de pensamiento, de una niña que iba al colegio del Sagrado Corazón, de una joven estudiante asidua de los centros neurálgicos de la política y la intelectualidad madrileña del momento $y$, pocos años más tarde, de una activa militante comunista. Pero, como afirma M. ${ }^{a}$ Dolores Ramos, "el eje cronológico no es el método natural de la memoria, más bien conviene considerar que cada sector de la vida ésta construido por sus propios materiales y que es el contenido temático el que permite identificar mejor los acontecimientos por orden cronológico ${ }^{6}$. Desde esta óptica, son dos los temas que marcaron el punto de partida, al que siguieron otros puntos de mira, en la trayectoria de Carmen: la educación y dentro de ella, la formación de la mujer en cuanto ser humano, con independencia de cualquier condicionamiento de género, en una sociedad que como ella afirma cestaba todavía muy lejos de plantearse la igualdad hombre-mujer".

Durante los años treinta, de forma intensa durante la República, en el ambiente de nuestro país y en el de muchas otras naciones, se palpaba fácilmente una honda preocupación por una falta general de educación. En este sentido habia muchas personas, entre ellas Carmen, que hicieron suyo el axioma de Leibniz: "dadme la enseñanza y cambiaré el planeta que habitamos" ?. Se consideraba la educación como primer factor de poten-

A lo largo de 1920 se produjo en el pais un desabastecimiento general de productos básicos, entre ellos harina. Esto ocasionó varias huelgas por parte de los panaderos y disturbios en las calles protagonizados por mujeres, con especial incidencia en Madrid y Barcelona. Vid. VVAA., Crónica del Siglo Xx. Madrid, 1986, vol. 1, p. 264.

6 RAMOS, $M{ }^{\circ}$. D.: "Pautas metodológicas para reconstruir la memoria histórica" en ALTED, A y AZNAR, m (eds.), Literatura y cultura del exilio español en Francia. Salamanca, 1998, p. 539.

Leibniz. Cit. por Samaniego, M., La política educativa de la Segunda República. Madrid, 1977 , p. 3. 
ciación en los distintos ámbitos del vivir, y su ausencia determinaba que social y económicamente a una gran mayoría de la población le estuvieran vedados unos valores de progreso y participación hasta entonces sólo al alcance de determinados sectores. Por lo que se refiere a la mujer, la aceptación de su inferior capacidad mental y la división tradicional de funciones según el sexo eran consideraciones interclasistas y aunque existían matizaciones explicativas en el caso de la izquierda, no obstante en estos sectores: anarquistas, socialistas, comunistas y marxistas heterodoxos, se encontraba con frecuencia la expresión de una duda sistemática sobre la capacidad intelectual de la mujer a pesar de sus reconocimientos oficiales acerca de la igualdad de derechos y deberes entre los sexos. Así, como afirma Carmen, el matrimonio era indudablemente "la carrera» más importante abierta a las mujeres a lo largo del periodo ${ }^{8}$. No obstante, durante los años de la Dictadura de Primo de Rivera, comienzan a surgir asociaciones de mujeres, sobre todo de clase media y con formación universitaria, que tratan de luchar contra esa situación. De las primeras en surgir fue el ya mencionado Lyceum Club, el cual existía en varias capitales, siendo fundado por primera vez en Madrid en el año 1926. Tenía por finalidad elevar el nivel cultural de la mujer, ser un lugar de encuentro y reunión, interesándose en la promoción de los derechos femeninos. A parte de sus secciones culturales, que organizaban conferencias, exposiciones, obras teatrales, a las cuales, como ya se dijo, asistía Carmen alguna vez, existía una sección oficial ocupada en estudiar y promocionar la igualdad legal de la mujer. Durante la República, el Lyceum y otras asociaciones que persegían idénticos fines ${ }^{9}$, adquieren mayor conciencia política, de cara a preparar a las mujeres para el ejercicio consciente de los recién adquiridos derechos políticos. En ese momento entre las socias del Lyceum se encontraban, la socialista Isabel $\mathrm{O}$. de Palencia y la republicana radical Clara Campoamor. No obstante, como reconoce Carmen, estas mujeres incluida ella, no eran representativas del resto de las españolas, en un pais en el que la mayoría de las familias pertenecian a un substrato social medio bajo, en el que el trabajo doméstico constituía la tarea fundamental, tanto de las mujeres casadas como de las solteras, y en donde además las elevadas tasas de paro daban prioridad al hombre al acceso laboral.

Vid. NASH, m., Mujer, Familia y Tabajo en España (1875-1936). Barcelona, 1983, p. 19.

Véase, Folguera, P.: "Las mujeres en la España contemporanéa» en Garrido Gonzalez, E (ed.). Historia de las mujeres en España. Madrid, 1997, pp. 417-564; Nunez PÉREz, M.` G., Trabajadoras en la Segunda República. Madrid, 1989, pp. 502-509; entre otras asociaciones estaban; la Asociación Nacional de Mujeres Españolas (ANME), la Liga para el progreso de la Mujer, la Sociedad Concepción Arenal de Valencia, la cruzada de Mujeres Feministas de Madrid, etc. 


\section{UNIVERSIDAD: LA FUE Y LA UFEH (1926-1931)}

Como se ha dicho, Carmen participó desde muy joven en las organizaciones que se encontraban a la vanguardia para trasformar la situación descrita. Su decisión de ir a la universidad "porque si mi hermano mayor había ido, pues yo también", fue determinante en este sentido, ya que la permitió entrar en contacto con personas, tanto profesores como alumnos, que estaban tomando conciencia del nuevo papel que la universidad debia desempeñar en la sociedad. No obstante, Carmen recuerda que por entonces, a sus diecisiete años: "no me planteaba como muchos problemas de futuro, porque no tenia mucha conciencia personal de cómo quería que fuese mi futuro". Cuando llega a la Facultad de Filosofia y Letras para hacer la especialidad de Historia, en el curso 1926-27, es el momento en que los estudiantes intentan establecer su influencia en la universidad para forzar la reforma educativa. Una de las organizaciones estudiantiles más activas fue la Federación Universitaria Escolar (FUE), fundada precisamente a comienzos de ese año a instancia de líderes estudiantiles tan significativos como Arturo Soria, ya mencionado, asi como Antonio M. ${ }^{\text {a }}$ Sbert, Antolín Alonso Casares y Emilio Gonzalez López ${ }^{10}$. Carmen entró en contacto con la asociación, en el acto inaugural del curso 1929-30, donde casualmente se sentó al lado de Soria, quien tras presentarse como uno de los dirigentes de la misma, le habló de sus fines y de la conveniencia de que se afiliara a ella. Lo que le dijo "me pareció muy sensato". Tanto es así, que su participación desde ese instante, en una organización fundamentalmente masculina: "en clase éramos ocho o diez chicas en un total de cuarenta", fue muy activa, llegando a formar parte de la junta directiva y siendo nombrada en 1930 secretaria general de la recién creada Unión Federal de Estudiantes Hispanoamericanos (UFEH) que buscaba una conexión con las organizaciones estudiantiles de Portugal y Latinoamérica. Con este propósito, el 17 de diciembre de ese mismo año, se celebró en México el Primer Congreso Iberoamericano de Estudiantes, el cual en 1931 tendría como sede Costa Rica, siendo elegida Carmen para asistir como delegada en representación de la universidad española. Es significativo de la situación de la mujer en aquel momento, no sólo en nuestro pais sino en el mundo, que ella fuera la única presencia femenina en dicho acontecimiento, y no sólo esto, sino que el día que tenía que pronunciar su conferencia: La Enseñanza Pública en las Escuelas, fue declarado fiesta nacional para los maestros, a fin de que pudieran acudir a

- Sobre la fundación de la FUE, Véanse, López-REY, J., Los estudiantes frente a la Dictadura. Madrid, 1930, pp. 26-28; GONZAlez LOPEZ, E., El espíritu universitario. Madrid, 1931, pp. 95-110. 
escucharla. Las líneas básicas de la misma fueron: enseñanza gratuita, mixta, escuelas en todas las poblaciones con un determinado número de habitantes, dotadas con profesores y medios estatales a fin de evitar el caciquismo..., "El discurso fue muy bien acogido. Los maestros asistentes decian: ¡ojalá, ojalá! A pesar del carácter excepcional que tuvo su intervención, ella recuerda haberla vivido "con toda naturalidad, porque siempre he estado integrada en el mundo masculino".

Volviendo unos años atrás, dado que se trata de una época esencial en la vida de Carmen, hay que decir que el objetivo primordial de las dos asociaciones era una reforma general de la educación inspirada en los principios de la Institución Libre de Enseñanza y unido a esto la necesidad de incorporar a los estudiantes obreros al mundo académico, objetivo inspirado en la Extensión Universitaria puesta en marcha en Zaragoza en 1893, aunque alcanzó su máximo apogeo en la universidad de Oviedo en 1898. Para conseguirlo tenian que obtener el control de la universidad por medio de su derecho a elegir el rector y decanos y también por medio de su representación en los órganos de gobierno de cada facultad.

En el testimonio de Carmen queda recogida, a través de su participación directa en ella, toda la reivindicación estudiantil y su recrudecimiento, debido a la transcendencia política que adquirió desde sus inicios. En concreto, recuerda con detalle uno de los actos de protesta que tuvo lugar en el $\mathrm{Pa}$ raninfo de la Universidad de San Bernardo: "consistió en tirar al suelo un busto del Rey y arrojar la cabeza a un canalillo de los Altos del Hipódromo". Como consecuencia de ello la policía detuvo a varias universitarias que habían presenciado los hechos, entre ellas Carmen, siendo encarceladas en la prisión de Quiñones de Madrid: “aquello constituyó una auténtica conmoción en la sociedad. Durante los días que estuvimos en la cárcel se acercaron cientos de personas a llevarnos flores y bombones. Al director de la cárcel le sobrepasó la situación. Para nuestra defensa se ofrecieron voluntariamente las abogadas Victoria Kent y Clara Campoamor» ${ }^{11 .}$

Los estudiantes se convirtieron en una de las principales fuerzas que contribuyeron a la caída de la Dictadura ${ }^{12}$. La dimisión de Primo de Rivera, el 29 de enero de 1930, había coincidido con una huelga estudiantil que derivó en

"Cir. Lopez ReY, J., op. cit., pp 237-246.

12 La causa concreta de conflicto universitario fue el art. 53 de la llamada reforma universitaria, llevada a la Gaceta por el catedrático Eduardo Callejo de la Cuesta de - aquí que se conociera como ley Callejo (1928) - quien a la sazón era titular del Ministerio de Instrucción pública y Bellas Artes. Sobre la lucha estudiantil, Véanse entre otros, lbidem, pp. 31-36; MONTERo HERNÁN$D E Z, E .:$ "El republicanismo de las organizaciones profesionales en la caida de la Monarquia en ToWNSON, N (ed), El republicanismo en España (1830-1977). Madrid, 1997, pp 332-338. 
la formación de un frente antimonárquico, al contar con el apoyo de grupos republicanos que tuvieron corta vida como la Asociación de Estudiantes Republicanos y la Liga Pro-unión y Acción Antimonárquica. El 4 de febrero, el Ministro de Instrucción Pública cerró las vacías universidades por treinta días. Con el fin de animar el ideal universitario de los estudiantes, mientras que la universidad permanecía cerrada, Soria ideó el proyecto de una Universidad Extraoficial o Universidad Libre. Proyecto que él mismo explicó a Ortega ${ }^{13}$, y que éste promovió con entusiasmo entre sus colegas, cuyos nombres están en la memoria de Carmen: Felipe Sánchez Román, Luis Jiménez de Asúa, Claudio Sánchez Albornoz, Agustín Millares, y que contó con la oposición de lo que ella, gráficamente denomina: "la caverna», en alusión a los profesores y alumnos integrados o próximos a los círculos de la derecha católica. Fueron muchas las instituciones que abrieron sus puertas y acogieron a profesores y alumnos. La Universidad Libre fue un gran éxito en todo el país y la acción más transcendental que los estudiantes habian llevado a cabo y demostró su capacidad para suplantar al Estado oficial en sus obligaciones. Además, la unión entre profesores y alumnos ofreció una visión de lo que podia llegar a ser la universidad. Durante todo este período, el Ateneo madrileño fue no sólo un centro estratégico de reunión universitario, sino uno de los puntos clave de agitación revolucionaria, donde se gestó día a día la llegada de la República. Como dice Carmen, asidua al mismo junto con Ricardo: "sólo con escuchar a los que allí hablaban, ya era suficiente".

Los que allí hablaban, eran entre otros: Valle Inclán, Baroja, Ortega, Unamuno, Azaña..., nombres que por sí solos bastan para poder imaginar la vitalidad cultural de un lugar en el que confluyeron a un tiempo miembros de la generación del 98 y de la generación del $14^{14}$ Carmen, desde su juventud de entonces, se refiere a ellos como "los mayores", y así dice: había una sucesión de pensamientos, los mayores sembraban [su palabra], entre los jóvenes". Otro lugar que frecuentará en ese Madrid de los años 30, "donde nos conociamos todos y que junto al Ateneo contribuyó a configurar una personalidad liberal, culta, e independiente, fue la Residencia de Estudiantes. Frente al "españolismo" del Ateneo, la Residencia tenía para Carmen: "otro aire". "Venia gente de fuera a dar conferencias", "por allí andaban Buñuel, Lorca, Dalí...". Se ha apuntado en varias ocasiones la amplia influencia de la Institución Libre de Enseñanza en las cátedras de universidad, institutos, normales, editoriales..., pero la principal fuerza del institucionismo radicaba en cuatro organismos de fina-

SORIA, A., op. cit. pp. 79-86.

JULIÁ, S., Manuel Azaña, una biografía politica. Madrid, 1990, pp. 76-79. 
lidad educativo-social en pleno auge en los años en que nos movemos: el Instituto de Reformas Sociales, la Junta para la Ampliación de Estudios, el Instituto-Escuela y la Residencia de Estudiantes. Esta última, desde su creación en 1910 hasta 1936, constituyó en el terreno intelectual, la tentativa más sólida de enlazar con el proyecto liberal y europeísta de cooperación y de intercambios internacionales que se fraguó, no sin resonancias políticas, en el mundo occidental de entreguerras. Fue el primer centro cultural de España y una de las experiencias más vivas y fecundas de creación e intercambio científico y artístico de la Europa de entreguerras ${ }^{15}$.

\section{AÑOS DE CAMBIOS, ESPERANZAS Y DECEPCIONES (1931-1936)}

En 1931 el país estrena República, su proclamación constituye un lugar común de la memoria. Carmen lo vivió asi: "yo estaba en el Museo de Artes Decorativas y el conserje vino a clase a dar la noticia. Salimos danzando locas, locas a la calle y nos encontrarnos con el pueblo entero. Nunca, nunca, se ha dado una explosión tan grande de alegría, que yo haya conocido, como la del advenimiento de la República. Fue la explosión de decir: esto lo hemos conseguido sin sangre, sin traumas gordos y con la aquiescencia del país, que ahi está. Estaba en la calle, todo, todo, todo el mundo. Hombres, mujeres, pobres, ricos, trabajadores, intelectuales". Pero hay un párrafo, en su testimonio, que sintetiza la esperanza de todo ese mundo "entonces no te planteabas cosas concretas de política. No había dado tiempo a plantearse nada. En ese momento, [la calle] era la expresión de un pueblo diciendo: ahora viene lo que es nuestro. No decias, ahora va a cambiar esto o aquello. No. Entonces tú decias: va a cambiar el país".

Uno de los cambios más ambiciosos que se propuso la Segunda República, en su objetivo de democratizar desde la raíz a la sociedad española, fue, dentro de la línea ya apuntada, la educación del pueblo en toda la amplitud posible. En relación a este tema, uno de los puntos que más polémica suscitó en los debates que tuvieron lugar en las Cortes Consti-

15 Sobre el significado de estas instituciones en el entomo cultural, politico y social de la época, Véanse, SANCHEz Ron, J.M. (coord.), La Junta para la Ampliación de Estudios e Investigaciones científicas 80 años después, 1907-1987. Simposio Internacional (Madrid, 15-17 de diciembre de 1987). CSIC, Madrid, 1988, vol. II. pp. 3-26, 201-238; PEREZ VILLANueva, I.: "La Residencia de Estudiantes en el Madrid de su tiempo" en VV.AA., Los origenes culturales de la I/ Republica. Coloquio de Historia Contemporánea de España dirigido por Manuel Tuñón de Lara. (9. ${ }^{\circ}$ Cuenca. 1992). Siglo XXI, Madrid, 1993, pp. 275-292. 
tuyentes de 1931 , a fin de redactar el nuevo texto constitucional, fue precisamente el de la enseñanza en conexión directa con la cuestión religiosa ${ }^{16}$, que, por otra parte, como ya se ha visto, ocupaba un lugar central en el proyecto transformador individual de Carmen. En este aspecto, dos eran las preocupaciones básicas de la izquierda: un sistema educativo que facilitara la movilidad social, y el fin de la escuela confesional. Corolario de estos planteamientos era la escuela única, gratuita y obligatoria.

Dentro de las realizaciones que se emprendieron destinadas a conseguir ese objetivo, y en conexión con el campo universitario, fueron las misiones pedagógicas, especie de nueva extensión universitaria llevada a escala nacional desde el Ministerio de Instrucción Pública con la colaboración especial de la universidad y a través del Museo Pedagógico. Una vez más la unión universidad —educación - transfórmación social entrará en el campo de actuación de Carmen, que participará en ellas, próxima ya a finalizar sus estudios de Historia. Estas misiones se componian de grupos de maestros y estudiantes con el material necesario para dar a sus auditorios obras de teatro, música en gramófono, y aún directamente ejecutada, cine, libros. En ellas trabajaron como misioneros personas que con su presencia marcaban lo que eran las misiones en lo que toca a su matiz ideológico: Cernuda, Salinas, Casona, José Antonio Maravall... Este experimento, verdaderamente creador, alcanzó gran éxito debido sobre todo a que se limitó a lo que permitían el personal y el material existente, y así, como recuerda Carmen, durante algún tiempo sólo íbamos a los pueblos de los alrededores de Madrid. Salías por la mañana muy temprano, porque los presupuestos no daban para alojamiento..., luego se fue mucho más lejos. Se llevaron libros y cine a pueblos donde la gente no había visto en su vida ni un libro, ni un aparato de cine y en esos libros mucha gente aprendió a leer». Confirmando sus palabras, Gabriel Jackson hace la siguiente valoración de las misiones: en muchos pueblos los campesinos jamás habían visto un automóvil, y mucho menos oído un fonógrafo o visto una película... En unos pocos días el contacto entre la Edad de Piedra y el siglo $x x$, entre campesinos analfabetos y estudiantes universitarios de la clase media, daba pocos resultados concretos. Pero se establecía un contacto vívido y sentimental de tremenda importancia potencial. Estos estudiantes habrian sido la futura generación gobernante de

16 Sobre el debate constitucional sobre la enseñaza y su implicación con la cuestión religiosa, véanse, Gi Pecharromán., La Segunda República. Esperanzas y frustraciones. Madrid, 1996, pp. 40-44; JULiA., S., op. cit., pp. 123-136. 
España si la guerra civil no se hubiera interpuesto, con su ingenuo y generoso deseo de enfrentarse a la pobreza, la ignorancia, el temor y la dignidad del pueblo español» ${ }^{17}$.

Por esas fechas comienza a trabajar, tras aprobar las oposiciones de auxiliar al Cuerpo de Archiveros, Bibliotecarios y Arqueólogos, [salida frecuente junto a la de la enseñanza, para las licenciadas en Filosofia y Letras ${ }^{18}$, en el Centro de Estudios Históricos, fundado el 8 de marzo de 1910 con el propósito de promover los estudios e investigaciones científicas. La dirección recayó en Don Ramón Menéndez Pidal. Estaba organizado por secciones, cada una de las cuales tenía a su vez su director o responsable de la organización de los trabajos y de los cursos que en ella se impartían. Eduardo de Hinojosa (Derecho), Claudio Sánchez-Albonoz (Instituciones Medievales), siendo en ésta donde Carmen continuará su formación como historiadora hasta el comienzo de la guerra. En su testimonio queda reflejado desde cómo transcurria una jornada de trabajo, hasta las relaciones existentes entre los miembros de la sección. Merece la pena recordar a los directores de las demás secciones: José Ortega y Gasset, que dirigia la de Filosofia contemporanea suprimida en 1916, Rafael Altamira (Historia y Metodología de la Historia), Americo Castro (Estudios Hispanoamericanos). Con tales maestros es fácil imaginar el ambiente de "familia intelectual" que debía respirarse en el Centro ${ }^{19}$.

Durante estos años no estará afiliada a ningún partido y organización, pero seguiría con preocupación los acontecimientos que se producen en el país, especialmente el incremento de la conflitividad social durante el verano de 1934, la progesión constante de los cedistas y agrarios hacia el control del Ejecutivo, y el freno que todo ello suponía para el proceso democratizador emprendido dos años antes. La revolución de octubre que tiene lugar en las cuencas mineras de Asturias y el norte de León y la dureza con la que fue reprimida llevaron a los sectores de la izquierda a corregir el rumbo de la República burguesa hacia la orientación revolucionaria a la que ya nos hemos referido. En este momento la trayectoria del país, y la de Carmen, es como si se diluyera en una sola. En el esquema que M. ${ }^{a}$ Dolores Ramos, siguiendo a Teresa Del Valle, propone como pautas para la reconstrucción de la memoria,

\footnotetext{
17 Jackson, G., La República Española y la Guerra Civil, Barcelona, 1976 p. 112.

18 Folguera, P., op. cit., p. 470.

19 Vid.Capitan Díaz, A., Historia de la educación en España. Madrid, 1994, pp. 462-464; Limon, E.: “El Palacio de Hielo: Sede del Centro de Estudios Históricos", en SánCHEz Ron, J. M. (coord), op. cit., pp. 605-622.
} 
aparecen las articulaciones, que son los momentos en que se adoptan decisiones, se producen encuentros y surgen respuestas a determinadas situaciones. Hay que adaptarse al medio, sopesar los apoyos, establecer el grado de consonancia o disonancia de las personas con los valores o expectativas del entorno ${ }^{20}$.

De acuerdo con todo esto, se puede decir que la Revolución de octubre constituye una articulación en la memoria de Carmen, quien ante lo sucedido decide colaborar en las actividades que, organizadas por el Socorro Rojo, trataban de ayudar a las familias de los represaliados. Aquí conocerá a Dolores Ibárruri, a quien desde entonces admirará incondicionalmente, como se desprende de su recuerdo hacia ella, y es probable que ese encuentro tuviera que ver con su decisión, unos años después, de afiliarse al PCE.

\section{EXPERIENCIAS DE GUERRA. LUCHA EN EL PCE (1936-1939)}

Retrocediendo un poco de tiempo, una noche del mes de julio de 1936, Carmen, tras haber estado por la tarde con Ricardo, al poco de llegar a casa, recibió su llamada desde la redacción de El Sol, comunicándole que un grupo de militares se habia sublevado en Africa. A la mañana siguiente, su familia, como tantas otras, estuvo pendiente de la radio. En medio de la confusión de aquellos momentos, continuó yendo a su trabajo, pero por poco tiempo, ya que una "nueva cotidianeidad" se iba apoderando de las calles y se iba imponiendo a sus habitantes, especialmente mujeres, quienes verían sus vidas transformadas por completo al tener que hacerse cargo al mismo tiempo del aprovisionamiento de sus familias y de aquellos sectores productivos y asistenciales que permitian sobrevivir a una ciudad sitiada ${ }^{21}$. Unas accedieron por primera vez al mundo laboral y otras, que ya estaban en él, tuvieron que reciclarse. Éste fue el caso de Carmen, que comenzó a trabajar en la Junta del Tesoro Artístico, órgano creado por el Gobierno republicano mediante Decreto de 23 julio de 1936, para proteger las obras de arte pertenecientes a museos, instituciones, iglesias y particulares que peligraban ante el constante bombardeo de la capital desde el inicio del conflicto. Su sede fue el Convento de las Descalzas Reales ${ }^{22}$.

20 Ramos,M. D., op. cit., p 548.

21 Ibidem., p. 518

22 "Al entrar en el Convento de las Descalzas Reales pudimos ver por nuestros ojos la razón de este Decreto (...). Nos demostró el verlas la multitud de enemigos que podian sumarse en su 
Decir que Carmen y su familia fueron marcadas por la guerra es no decir nada, pero cuando se escuchan los testimonios de quienes la sufrieron, se llega a la conclusión que en España hubo tantas guerras civiles $y$ todas tan distintas, $y$ al mismo tiempo con tantos lugares comunes $y$ sentimientos compartidos, que cada una de esas experiencias merece ser recogida y de forma exclusiva. En este sentido, los recuerdos de Carmen Caamaño son tan vívidos y están contados con tanta coherencia y lucidez que resulta obligada la remisión a ellos. No obstante, y a efectos de continuar definiendo las coordenadas que sirven de marco espacial y temporal a su trayectoria, hay que decir que con el traslado en noviembre de 1936 del gobierno a Valencia, ante el inminente ataque de las columnas sublevadas, Carmen comenzó a trabajar en esta ciudad, en la Subsecretaria de Educación que estaba a cargo de Wenceslao Roces, siendo Jesus Hernández, titular de la cartera y que, junto a Vicente Uribe de la Agricultura, fueron las dos incorporaciones comunistas al gobierno que Largo Caballero había constituido el 4 de septiembre de ese mismo año dentro de las alianza definitiva de todas las fuerzas prorepublicanas contra el fascismo en un intento de dar un sentido político enteramente nuevo a la defensa de la República y al régimen mismo ${ }^{23}$.

A partir de 1937 se inicia una etapa esencial en la vida de Carmen, tanto en el aspecto personal (contrae matrimonio con Ricardo), como el político, ya que, como se ha dicho, se afilia al Partido Comunista de Alicante, ciudad a la que se trasladaron recién casados. En realidad fue como hacer públicos dos compromisos que habian sido tomados tiempo atrás por lo que se puede afirmar que este periodo representa, no un punto de partida, sino un punto de mira más dentro de su trayectoria, si bien es cierto que: "mi proyecto político se definió con la guerra». En Alicante trabajará en la secretaría de la Mujer del Partido y posteriormente como secretaria personal del Gobierno Civil, Jesús Monzón ${ }^{24}$, camarada y amigo, de quien al igual que Dolores, recuerda como ejemplo de honradez y de entrega a una causa, al margen de ambiciones personales. En palabras de Carmen "hacian una labor de tiempo de guerra y no de aprovechamiento de guerra"... En concreto, su tra-

posible destrucción: el fuego, la metralla, el derrumbamiento, el agua, el frío, el desconocimiento de su valor (...) y los hombres encargados de su salvaguardia se veian desbordados de trabajo continuamente». Vid. León, M. ' T., La historia tiene palabra: noticia sobre el salvamento del Tesoro Artistico. Madrid, 1977, p. 40.

23 Vid. Arortegui, J., La Guerra Civil, 1936-1939, Madrid, 1996, p. 67

Sobre la trayectoria de Jesús Monzón en la dirección del PCE en el exilio Francés Vid. EsTRUCH TOBELla, J., El PCE en la clandestinidad (1939-1956), Madrid, 1982, pp. 85 y ss. 
bajo, una vez más, estará relacionado con la formación de las mujeres, a fin de que pudieran desempeñar con eficacia y "con consciencia de la importancia de su labor" una serie de funciones, "hasta entonces en manos de hombres", para mantener la productividad tanto de las industrias de guerra como del campo en una zona tan estratégica para el abastecimiento de la República como era Levante. A finales de 1938 la vida de Carmen discurrirá tan precipitadamente como los sucesos que marcaron ese periodo dramático de nuestra historia. Monzón es destinado a Cuenca, también como gobernador, y al poco tiempo, debido a la situación crítica de la guerra, marcha hacia Madrid. Apenas había tiempo de buscar alguna persona que se hiciera cargo del puesto que él dejaba vacante, y decide, con el visto bueno del Partido, que la persona que mejor puede desempeñar ese cometido es Carmen, que estando embarazada, decide aceptar. En su memoria esta situación aparece recogida como una encrucijada o como una intersección ${ }^{25}$, estableciendo la línea divisoria entre lo que se hizo y lo que se dejó de hacer. Pero como ella acostumbra a decir "a mí no me asusta la responsabilidad. Yo sólo quiero saber cúal es la realidad y una vez que la sé, me instalo en ella". Con la entrada de los franquistas en Cuenca, Carmen partirá hacia un lugar común de la memoria, el puerto de Alicante, esa franja de tierra, única ya de la que fuera la España republicana, a la que miles de personas se dirigieron, con la esperanza de poder tomar un barco en el que poder salir. Tal era el caso de Carmen. Sin embargo, cuando iban a subir al barco, el último que entró al puerto, fue tal la avalancha de refugiados que intentó subir a él, que el capitán, ante el riesgo inminente de hundimiento, tal y como recuerda Carmen: "quitó la escalerilla y dijo: ¡nadie! Y nos dejó alli. "Regresó a Alicante, a casa de un amigo médico, y allí a las pocas horas dió a luz. Al día siguiente volvió al puerto con el niño, pero no volvió a entrar ningún barco. Los italianos y falangistas habian rodeado el recinto portuario con ametralladoras, convirtiéndolo en un campo de concentración ${ }^{26}$. "Cada vez llegaba más gente al puerto y entre ellos aparece Ricardo (...). cuando nos encontramos pensé: por lo menos lo que sea de uno va a ser de los dos".

\section{LA ZONA GRIS DE LA MEMORIA (1939-1947)}

A partir de aquí, comienza lo que Carmen denomina La zona gris de la memoria, plagada de vivencias, "que se sabe que están ahí, pero que

25 Ibidem.

26. Vid. Dona, J., Desde la la noche y la niebla (mujeres en las cárceles franquistas), Madrid, 1978. p. 56 
fueron tan poco constructivas para mi como ser humano", que siente desgana al pensar en ellas. Aun así, prefiriendo no incidir en los recuerdos más dolorosos, aunque sin soslayarlos en ningún momento, lo hace de forma detallada y serena, unas veces dejando entrever un cierto orgullo, no sólo por haber sobrevivido a ellas, sino porque no consiguieron cambiar en ningún momento las razones de su lucha ni de su compromiso político. Sin embargo, en otras, la explicación puede encontrarse en un carácter indomable que se niega a admitir el daño que le hicieron.

En Carmen, como sucede en otros testimonios, los recuerdos no son del todo puros, hay una mezcla de lo vivido y de lo asimilado a posteriori. En su caso es evidente el interés por enterarse de la interpretación histórica de los hechos, con el objeto de someter lo vivido a un proceso de racionalización a fin de encontrar respuestas a preguntas entonces incontestables, siendo esto frecuente en situaciones históricas extremas como el caso de una guerra civil ${ }^{27}$. Así, hace poco ha concluido la lectura de la biografía política de Manuel Azaña ${ }^{28}$, y refiriéndose a este libro dice: "me ha gustado porque me ha dado el porqué a muchas cosas que entonces no entendía". En los momentos más duros de esa experiencia hay en su testimonio una frase que se repite: «ya nada me importaba porque habíamos perdido la guerra", pero a continuación aparece de nuevo, con la espontaneidad de un acto reflejo, su inagotable capacidad de lucha, y así, en pleno abatimiento físico [a consecuencia del parto sufria una fuerte hemorragia], recuerda cómo en el mismo puerto, cuando la desesperación inducía a muchos al suicidio ${ }^{29}$, trataba de animar y explicar a las mujeres que se hallaban a su lado, y que al igual que ella estaban desoladas, que la lucha no había sido en balde y que había que seguir adelante. En este punto, el hilo del relato queda interrumpido en varias ocasiones por lo que a su juicio fue una causa fundamental de la derrota y de la duración de la contienda, la ayuda extranjera y el abandono en que dejaron a la República las naciones democráticas, dicho con sus palabras: «lo de la ayuda internacional, fue una vergüenza".

Al poco tiempo se iniciará en su vida y en la de su familia, como en tantas otras, una época marcada para siempre por una represión cruel e irracional, tanto la que tuvo lugar en los primeros años de victoria como durante la larga postguerra. Sesenta años después, el testimonio de Car-

27 EGIDO, A.: “Eslabones del Quinto regimiento. las milicias regionales. Experiencias u reflexiones de un capitán del Batallón segoviano" en ALTED, a. (coord), Entre el pasado y el presente. Historia y memoria, p. 133.

28 Carmen se refiere a la obra de Santós Juliá ya citada.

29 Vid. Dona, J., op. cit., pp. 53-59. 
men no es una mera descripción de acontecimientos, sino un ánalisis lleno de lucidez, a veces de tristeza, y vacio de rencor. Carmen fue detenida, juzgada y en dos ocasiones. La primera de ellas junto a Ricardo y a su hijo recién nacido, en el pueblo de San Juan, tras haber conseguido escapar por puro azar de las tropas franquistas que, como se ha dicho, habian tomado el puerto. En esa ocasión, fueron ambos condenados, a 12 años y un día de presidio mayor, "por ser ese matrimonio de la Institución Libre de Enseñanza", lo que es bastante significativo no ya sólo de la arbitrariedad legal y judicial del nuevo Régimen, sino también de lo que comenzaba a ser la formación del espíritu nacional de sucesivas generaciones de españoles. A consecuencia de un indulto general ${ }^{30}$, debido a que como dice Carmen: "las cárceles estaban a rebosar", ambos salen de sus respectivas cárceles de Alicante en 1941. De esos años, en la zona gris de la memoria quedan muchas vivencias, entre ellas dos: la separación de su hijo recién nacido y la amistad de la compañera con la que compartía diariamente un trozo de suelo de una inmensa nave, unas lentejas llenas de cristales, y las últimas horas antes de que la separaran de su lado para ser fusilada.

En la siguiente ocasión, sólo la detuvieron a ella. Junto a otros militantes, entre ellos Ricardo, Carmen intentaba organizar el Partido Comunista, en la clandestinidad, en la zona de Alicante. Al igual que habia sucedido en otros partidos, los cuadros intermedios y militantes de base del PCE: "habian quedado abandonados a su propia suerte" ${ }^{31}$. A pesar de ello, no se resignaron a permanecer totalmente inactivos. Los primeros intentos de organización clandestina del PCE fueron del verano de 1939, con José Cazorla y Enrique Sánchez, que acabaron en el paredón, pero dejaron un comité provincial en Madrid del que formaba parte el dramaturgo Buero Vallejo. Hasta 1941 no empezó la oposición seria. En el mes de mayo de ese año el PCE intentó crear en Portugal una organización estable, conocida como Operación Lisboa, que iría acompañada de otra paralela en España. En concreto en Madrid la dirección estaría a cargo de Heriberto Quiñones y Luis Sendín. En septiembre de 1941 se produjo en nuestro país una redada que ocasionó numerosas detenciones, al tiempo

30 Aunque Carmen no recuerda la fecha del indulto, el 1 de abril de 1941, fueron puestos en libertad 40.000 prisioneros en un indulto general promulgado para celebrar el segundo año de la victoria franquista. Vid. PAYNE, S. G., El primer franquismo, 1939-1959, los años de la autarquia, Madrid, 1996, p. 14.

31 Estruch Tobella, J., op cit., p. 11. En el sentido, Tomasa Cuevas militante comunista recuerda que: "puede decirse, porque asi fue, que hasta el año 1942, fecha de mi detención [...] anduve como tantos y tantos a salto de mata". Vid CuEvas, T., Cárcel de Mujeres, Barcelona 1985, p. 126 . 
que en Portugal el grupo de Lisboa era detenido por Salazar y entregado a Franco. Entre los detenidos en España se encontraba Quiñones que, tras ser sometido a incontables sesiones de tortura en la Dirección General de Seguridad, fue fusilado atado a una silla por estar completamente destrozado, el 2 de octubre de $1941^{32}$.

A consecuencia de todo ello, en Alicante también cayó la incipiente organización. Entre los detenidos estaba Carmen. Este es su testimonio de lo sucedido: "el Partido me encargó la reorganización en esa zona [Alicante], pero en la clandestinidad todo era muy distinto. La organización se basaba en una cadena de pequeños grupos, tres personas, las células, de las que sólo una comunicaba con la siguiente, para en el caso de detención, no poder decir nada por mucho que te torturaran. Yo comunicaba con una persona en Madrid, y esta fue detenida con todas las direcciones y nombres de la organización en Levante. Le fusilaron en la misma silla en la que había sido torturado, y claro vinieron a buscarme a casa... y ihala!, otra vez".

El juicio de Carmen y el de sus compañeros, que tuvo lugar en Alcalá de Henares, fue el primero que celebró el Tribunal para la Represión de la Masonería y del Comunismo, creado por la Ley de 1 de marzo de 1940, promulgada en complemento de la de 9 de febrero de 1939 de Responsabilidades Políticas. El comunismo - un término que se aplicaba de forma general a toda la izquierda radical y revolucionaria- era el enemigo político número uno. Para Carmen y sus compañeros, el fiscal habia solicitado la condena a muerte. La sentencia ratifico esa petición para todos, excepto para Carmen, que fue condenada a 20 años y un día. Fue trasladada a la cárcel de Ventas, que con la de Claudio Coello y Quiñones, eran las cárceles de mujeres que había en Madrid. Desde el primer momento, las reclusas comprendieron que su única salvación era no perder su espiritu militante. Que el terror de la cárcel había que hacerle frente mediante la organización. En este punto el testimonio de Carmen es significativo: "en la galería, casi todas éramos del Partido menos seis que eran socialistas. Teníamos, y entrecomillo "reuniones políticas" ${ }^{33}$, que consistian en que a través

\footnotetext{
32. Vid Moreno, F.: "La represión en la posguerra" en Julia, S. (coord), Victimas de la guerra Civil, Madrid, 1999, pp. 277-423.

33 El testimonio de Carmen lo confirma Tomasa Cuevas militante comunista y presa en Ventas durante el periodo 1942-1946. Lo mejor de la prisión era la maravillosa organización del Partido, que te hacia sentirte plenamente feliz por cuanto alli se trabajaba. Las presas políticas no queriamos convertir el periodo de reclusión en tiempo perdido, sino de provecho, y con este fin se organizaron grupos de estudios, de formación política, de cultura general; había una gran actividad política, la discusión de periódicos que caían en nuestras manos y algún que otro material del Partido. Todo ello comportaba un riesgo" Cuevas, T., op. cit., p. 152; Vid. También Ibidem. p. 81.
} 
de la prensa que se conseguía entrar en la cárcel, te enterabas de la situación que había en el gobierno y comentábamos lo que pasaba en España y en el mundo... Sabíamos que el Partido seguía funcionando en la clandestinidad porque las familias de muchas presas estaban trabajando políticamente y cuando iban "a comunicar» ${ }^{34}$, contaban lo que sucedia. Se sabía que había una resistencia en las fábricas y un frente antifranquista. En aquel momento todo el objetivo era ver cómo se luchaba contra Franco, cada uno desde su puesto de trabajo, sabotajes los que estaban en sitios que podían hacerlo, los que podian escribir algo para orientar escribian, todo tenía su riesgo porque los muertos llegan hasta casi cuando Franco se muere, pero la gente siempre ha creído mucho en las cosas. Precisamente, por ser sorprendida leyendo uno de esos periódicos clandestinos, Carmen pasaría una semana en una celda de castigo y los últimos cuatro meses de condena, en la cárcel de presas comunes de Cáceres, como dice con sentido del humor: "aquello fue la guinda", "las presas de alli no me consideraban una de las suyas". Volvió a pisar la calle, que no a estar en libertad, en 1947 tras cumplir las tres cuartas partes de la condena.

Había pasado en la cárcel un total de siete años de los que extrae este balance: "no es una cosa que me encante contar. Muchas veces lo recuerdo, y muchas veces pienso en ello,-, pero es algo,-, que no es una cosa que yo me esfuerce porque esté, he intentado dejarlo en gris, porque no era nada constructivo, era una situación que no te estaba formando en ningún aspecto, ni servia para enriquecerte como persona,--, es como una laguna en tu vida". Aun así, los vínculos de amistad con algunas de sus compañeras amortiguan lo anterior: "uno se acostumbra a todo...», "no tengo tan poco tan mal recuerdo, porque como era gente muy estupenda con la que estabas, vivias como fuera del mundo" ${ }^{35}$.

34 En el argot de la cárcel significaba, recibir visitas de familiares y amigos: "Un pasillo ancho separaba a las reclusas de sus visitantes. Gritaban todos al mismo tiempo. Vistos desde tucra parecian locos. Tanta desesperación habia en sus voces para hacerse oír». Ibidem, pp. 144-145: «El día de comunicación con el exterior era un día de fiesta para las que tenian la suerte de ver a su familia, pero también de tristeza para aquellas que estaban alejadas de los suyos por muchos kilómetros de distancia. Las noticias transmitidas a través de las comunicaciones no eran muy alegres, casi siempre llegaba alguna de la brutal represión a que estaba sometida la gente, fusilamientos, detenciones. A decir verdad fueron los célebres bulos de la familia que nos infundian la esperanza de un fin inmediato de aquella situación, lo que nos ayudó mucho a no caer en estado de desesperanza". Ibidem.

35 La salida de Ventas fue muy emotiva, pues eran años de permanencia en la misma y allí quedaban grandes camaradas $Y$ entrañables amigas con las que habiamos pasado amarguras $y$ también las pequeñas satisfacciones que alli recibiamos". lbidem, p. 126. 


\section{EXILIO INTERIOR. OPOSICIÓN AL RÉGIMEN (1947-1975)}

Tras esos años de cárcel, Carmen se encontró con una dificil situación: "tardas mucho en instalarte, en que la cabeza se reinstale en otra vida diferente. La cabeza sigue en Ventas, donde has dejado a gente con la que has convivido muchas horas. Entonces no te instalas en un sitio ni en otro ... Sales y piensas que hay que buscar un trabajo, que tienes que ayudar en casa porque las cosas son muy difíciles económicamente, y en tu hijo, al que no has visto durante años, salvo esporádicas visitas a la cárcel en las que me daba cuenta que ya no me conocía, que éramos dos extraños. Se había quedado en Alicante en casa de unos amigos, iporque vete a explicar a un niño que no hay comida ...! ?. En ese momento Carmen y Ricardo compartían una casa situada en la Travesía de San Mateo con la madre, las hermanas y los sobrinos de éste: "viviamos con 500 pesetas al mes". Profesionalmente estaba sujeta a un expediente de depuración, que hasta los años sesenta no recuerda la fecha exacta le impediría volver a su puesto de trabajo en el Centro de Estudios Históricos.

Sin embargo, un amigo le proporcionó un trabajo: "tenía un amigo en Estados Unidos, José López Rey, que era catedrático de Historia del Arte en Estados Unidos ${ }^{36}$. Yo le proporcionaba, buscando en archivos, la documentación que sus alumnos necesitaban para, por ejemplo, el estudio de un pintor español del siglo XVII, y eso me daba un dinero."

El proceso de adaptación fue extremadamente difícil por la represión que sobre ella, como sobre otros muchos, ejercia el Régimen "yo tenía que presentarme a la Policia, primero cada quince días, y luego cada mes. Así estuve durante mucho tiempo... No hacias una vida espontánea tuya. Estabas siempre pensando que la Policía andaba detrás. Yo estaba permanentemente seguida. Un día llegué a casa y me dijo el portero que había estado la Policía, y yo le pregunté: ¿qué te querían?, y me contestó: "a mí nada. A usted". En otra ocasión se presentó la Guardia Civil: "fue después de un viaje que hicimos a Galicia... Vinieron a preguntarme que para qué había ido y a quién habíamos visto, y entonces yo contesté: lo saben ustedes muy bien, porque donde yo he ido han venido ustedes detrás de mì.

36 José López-Rey fue uno de los fundadores de la FUE y autor de la obra Los estudiantes frente a la Dictadura. Vid $n .^{\circ} 10$. 
De su padre y hermanos sólo sabía a través de un amigo francés que estaban en Francia. Ese amigo había conseguido, tras una intensa búsqueda, sacarlos de los distintos campos de refugiados en los que habian sido recluidos. Poco a poco rehicieron su vida en Marsella. Carmen sólo obtuvo permiso oficial en dos ocasiones para ir a ver a su padre, la última antes de morir éste. Durante muchos años la única comunicación con ellos consistía en: "unas cartas anodinas, porque sabias que iban a ser leídas, diciendo: estamos bien, vivimos así ... Y ellos igual: estamos bien y hacemos esto..." Su padre, Pedro, no quiso volver mientras estuviera Franco. Para él, como dice su hija: "era una cuestión de principios. Echaba mucho de menos España. No se llegó a adaptar a la vida francesa nunca».

Debido a este seguimiento permanente no podía participar en las actividades que llevaba a cabo el Partido en la clandestinidad. No obstante, mantenía contactos con militantes activos que le informaban de las directrices y estrategias de la organización al hilo de los acontecimientos que tenían lugar en el país. Sin embargo, conforme avanza el tiempo, Carmen volvería a implicarse directamente en ellos, y de nuevo será la universidad la que le proporcione la dirección a seguir. Por el seguimiento al que era sometida, su cotidianeidad transcurría dentro de un círculo muy reducido: "me limitaba a las amistades de antes. En el trabajo [una vez que dejó de estar inhabilitada] yo no tenía una relación ni de intercambio de ideas, ni de intercambio de afectos. Limitabas tu círculo a un "grupitín" muy pequeño de gentes "anteriores a...", y lo otro eran conocidos circunstanciales con los cuales mantenías una relación digamos, de buena educación y de intranscendencia de pensamiento". "Antes de emitir un juicio mirabas mucho, porque no estabas seguro de cómo iba a ser la reacción de el de al lado". A pesar de esto, y a medida que el control policial sobre ella iba disminuyendo, Carmen reanuda su actividad militante de acuerdo con lo que iban dando de sí las circunstancias: "cuando el Partido empezó a funcionar un poco a la luz del día, yo iba a todas las reuniones de la agrupación de cultura y a las demás que me interesaban. Empecé a seguir la política más directamente». El contexto socio político comenzaba a experimentar cambios significativos, entre ellos, por su específica repercusión en la trayectoria de Carmen, es preciso destacar los siguientes: desde 1948 aparecen en Madrid centros de encuentro y contactos de carácter más liberal con visos de oposición. Fueron el Ateneo, desde su reapertura en 1946, el Liceo Francés, algunos cafés y Círculos Recreativos ... En todos estos centros, así como en los colegios mayores universitarios, tanto de la Iglesia como del Estado, se fueron encontrando jóvenes estudiantes, po- 
etas, intelectuales procedentes de muy diversas familias y clases. «Pensar bajo el franquismo se convertía más que en un reto en una ansiedad, tanto más profunda, amplia e inconcreta cuanto más avanzaba uno en la transgresión de la norma prohibitiva, norma que casi todo lo cubría y que en todo depositaba suspicacias" ${ }^{37}$. Se trata de años de intensa actividad creadora y de inquietud social y política que obliga a la reflexión y al cambio de mentalidad entre los sectores, dirigentes y responsables de la época. A partir de 1952, la apertura al exterior, los contactos internacionales, el descubrimiento del exilio como una realidad insoslayable, van constituyendo facetas y aspectos de una situación cada vez más compleja y difícil. De este modo, «Hubo un exilio interior que propugnó por dejarse oír, opuso una resistencia pasiva o trató de sobrevivir quedamente frente a un régimen que utilizaba la represión física y la censura cultural como instrumentos de poder" ${ }^{38}$. A pesar de ello, esta oposición interior a medida que pasa el tiempo adopta estrategias cada vez más activas y contundentes. De nuevo el papel de los estudiantes, como parte del grupo social más amplio, el de los jóvenes en general, será determinante, alcanzando su cenit, en cuanto a su repercusión en la política nacional, en 1956, año en el que los disturbios en las universidades de todo el pais, pero principalmente en la de Madrid, provocaron la primera crisis grave a la que hubo de enfrentarse el régimen. A la vista de los acontecimientos la dirección del PCE percibe que es en el terreno universitario donde las estrategias de la lucha arrojan a corto plazo más y mejores resultados que las llevadas a cabo hasta ese momento en otros ámbitos, en concreto el sindical. A partir de esa fecha, el Partido desplegará una intensa actividad en medios universitarios e intelectuales, todo ello dentro de una nueva política de reconciliacion nacional y teniendo entre otros objetivos aproximarse a las demás fuerzas de oposición a fin de romper el aislamiento que respecto a estas mantenía desde la posguerra.

Dentro de esa coyuntura el 17 de noviembre de 1953 un grupo de mujeres funda en Madrid la Asociación Española de Mujeres Universitarias que según el artículo primero de sus estatutos ${ }^{39}$, aspiraba a ser

37 HeRníndez SandoICA, E.: “Universidad y oposición al franquismo. Reflexiones en torno a los sucesos de 1956 en Madrid." en TuselL, J; ALtED, A y MATEOS, A. (coords), La oposición al regimen de Franco, 1990, tomo II, p. 188.

38 ALTED, A., prólogo a CAUDET, F., Hipótesis sobre el exilio republicano de 1939 madrid, 1997, p. 11.

39 La documentación relativa a esta asociación se encuentra en la Fundación José Ortega y Gasset de Madrid. Los datos que se ofrecen aqui, han sido obtenidos personalmente del libro de actas. El resto de la documentación se halla sin catalogar. 
incorporada (como así fue) a la Internacional Federation Of University Women de Landvel. Entre sus mienbros estaban: Jimena Menéndez Pidal, Isabel García Lorca, Pilar de Madariaga, Soledad Ortega, Laura de los Ríos, Natacha Seseña,... y Carmen Caamaño, entre muchas otras, que a lo largo de más de treinta años de vigencia de la Asociación contribuyen activamente a que se hiciera realidad su finalidad esencial dentro de las directrices de la federación internacional que era "la lucha por las obras sociales y asuntos públicos». Este objetivo, de largo alcance, se concretaba dentro de la realidad española esbozada en las líneas anteriores en una intensa actividad cultural que como matiza Carmen: "en esa época la cultura siempre acababa en política". Las actividades organizadas en el local de la asociación, en la calle Miguel Angel $n .{ }^{\circ} 8$, o en cualquiera de los cedidos por diversas instituciones, debian contar con la debida autorización de la autoridad gubernativa y, dado el contenido "cultural" de muchas de esas actividades, los problemas surgian a menudo. En una ocasión se produjo el cierre de la sede durante tres meses. Basta con mirar el libro de actas donde están recogidas todas las asambleas generales para confirmar este extremo. A título de ejemplo alguna de las actividades previstas para 1968 fueron entre otras: "cursillo sobre Marx con ocasión del 150 aniversario de su muerte». Julián Marías, José Luis Sampedro, Santiago Montero, Aranguren...fueron algunos de los intelectuales que dieron conferencias contando con un número cada vez más numeroso de asistentes, especialmente universitarios, y también, como recuerda Carmen: "siempre se encontraban un par de policías". Su actividad en la asociación la resume diciendo que: "yo estaba en todo y en nada...". Dado que estaba condenada penalmente a inhabilitación para cargos públicos, no podía desempeñar ningún papel directivo. Esta es la razón por la que a través de las actas mencionadas se comprueba que lo mismo estaba organizando para el cine-club un ciclo de temática feminista: "en un país [en el que como ella dice], la mujer habia vuelto a ser la portada de los valores eternos", que proponiendo un ciclo de conferencias sobre la Institución Libre de Enseñanza, o diciendo que el Padre Llanos le había dicho que necesitaba gente para dar clases en el «Pozo del Tío Raimundo". A partir del 19 de mayo de 1989 no consta en libro de actas ninguna actividad más de la Asociación.

Antes de esa fecha habian tenido lugar tres acontecimientos concatenados muy importantes para el país y para Carmen: la muerte de Franco, la llegada de los exiliados y la legalización del PCE. Estos dos últimos supusieron un encuentro con una parte esencial de su pasado. Recuerda en especial la llegada de Claudio Sánchez-Albornoz en 
1976 40: "Yo estaba en el aeropuerto cuando llegó, pero fue asi, así porque no hubo comunicación. Luego cuando enfermó fui a verle al hospital en Ávila. Fueron las últimas veces que le vi. Ya no contaba nada porque físicamente estaba muy mal». También la llegada de Dolores en $1977^{41}$ : "tuve un trato muy cordial con ella cuando llegó de la URSS (...). Tenía una fuerza, una personalidad extraordinaria, pero al mismo tiempo con una ternura, dulce, dulce ,-, Dolores era como una abuelita, era un ser excepcional". "A la hora de ser enérgica no había persona que lo fuera más, ni hombre, ni nadie ,-, porque todo el mundo respetaba a Dolores". "La gente decía: Io ha dicho Dolores...". Uno de esos encuentros entre ambas tuvo lugar en el primer mitin del PCE. Tras su legalización en abril de 1977, en la primera campaña electoral de una incipiente democracia: "eso lo viví de una manera emocional tremenda. Era la presentación oficial del Partido. Diluviaba. Nos volvimos a casa. Dejó de diluviar y volvimos. Para mí volver a ver las siglas del PCE, a los camaradas que no había visto durante tanto tiempo, ver a gente joven incorporada a todo eso - para mí fue una cosa- que no se me olvidará...". "Lo viví con toda la ilusión, que no duró mucho (...) porque los poderes fácticos seguian siendo los mismos..., porque en España nunca han dejado de mandar la Iglesia y el dinero,-, nunca, nunca, nunca, en ningún momento».

Resulta especialmente significativo como Carmen analiza el regreso de los que se fueron, desde su mirada y desde su experiencia en el exilio interior. Para ella lo que se produjo fue un auténtico desencuentro: «Nunca llegaron ya a sentirse a gusto cuando regresaron. Estaban ya influenciados por demasiadas cosas externas a lo que era el pais y su integración, a mi modo de ver, nunca la hicieron completa (...). En mi generación nunca ha habido una compenetración entre los que se quedaron con los que se marcharon. Había un algo...". "Hay una cosa indiscutible tu no puedes juzgar el franquismo si lo has vivido aquí, que si siendo español lo has vivido fuera. El juicio no puede ser el mismo (...). Habia siempre una cierta cosa, como de... si claro, pero tú no estabas cuando..." "Ellos siguieron los acontecimientos a través de..., pero no los vivieron. Hay una cierta diferencia entre vivir directamente una situación a recibirla como noticia. Al cabo de los años eso va dejando un poso que a mí me parece que no desaparece. Cada uno intenta darse razones para decir: yo hice lo que tenia que hacer, aunque puede que

40 Vid. Pamies, T., Los que se fueron, Madrid, 1976, pp. 173-178.

4t Vid. Pámies, T., op. cit., pp. 187-189. 
no fuera así». Carmen recuerda por ejemplo, los viajes a España de su amigo José López-Rey, durante la dictadura: "él no venía como español, venía como norteamericano y eso hacía que las cosas para él fueran diferentes (...) Yo creo que en lo del exilio no se puede generalizar. Cada caso es completamente distinto a los demás y resultado de muchas circunstancias (...) Mi hermano Antonio era en España registrador de la Propiedad y en Francia tuvo que trabajar de carbonero para ganarse la vida...».

\section{EL PRESENTE}

En esta breve historia de vida de Carmen Caamaño se ha recogido sus años de infancia, la juventud y sus largos, intensos y productivos años de madurez. Pero quedaría incompleta y sería una torpeza no hacer ninguna referencia a su vejez, porque es en esta etapa donde muchas personas, Carmen entre ellas, llevan a cabo una lucha silenciosa con la que culminan una existencia vivida con plenitud y en la que se condensa toda la conciencia y la dignidad que les ha acompañado durante muchos años. Hay una frase que en la actualidad repite con frecuencia: "yo ya no tengo vida". Sin embargo, cuando se han compartido con ella unas pocas horas de su jornada diaria sorprende que su interés por lo que la rodea siga siendo tan intenso. Lee incansablemente periódicos y libros: "aunque me han dicho que sólo lea con luz natural", y en el restaurante donde come desde hace mucho años se convoca todos los días, de forma espontánea, en su mesa, una pequeña tertulia de asiduos al local, donde se pasa revista a lo sucedido en las últimas horas. En esas conversaciones Carmen continúa mostrando el mismo inconformismo y continúa indignándose por las mismas cosas por cuyo cambio ha luchado durante toda su vida, y sostiene entre risas que: "seguimos bastante cogidos por el "pescuecito", por los mismos que nos han tenido cogidos siempre...". En este sentido, ha sido y es una firme referencia para todas las personas -especialmente mujeres-que hemos tenido el privilegio de conocerla.

La última parte de su testimonio es reflejo de una persona que ha sabido ser siempre fiel a sí misma: «En ningún momento he pensado que aquello en lo que me he metido a lo largo de mi vida no ha valido la pena. Lo hice pensando que era mi obligación, porque si no, no me iba a quedar a gusto conmigo misma". "En ningún momento fui empujada, o porque me hicieran ver las cosas. Siempre he sido yo la que he decidido y nunca me he planteado mucho el por qué" "Siempre me ha 
parecido que la vida me ha ido diciendo dónde debía poner los pies, y no me he planteado si tenía que poner primero el derecho antes que el izquierdo. Sabía que tenía que caminar con los dos a la vez".

\section{FUENTES Y BIBLIOGRAFIA CONSULTADAS}

Fuentes

Fundación José Ortega y Gasset: Libro de Actas de la Asociación de Mujeres Universitarias Españolas.

\section{BIBLIOGRAFIA}

ALTED, A (coord.): Entre el pasado y el presente. Historia y memoria, Madrid, UNED, 1995, $182 \mathrm{pp}$.

ALTED, A Y AzNAR, M (eds.): Literatura y cultura del exilio español en Francia, Salamanca, AEMIC-GeXEL, 1998, $550 \mathrm{pp}$.

Arostegl, J.: La Guerra Civil 1936-1939. La ruptura democrática, Madrid, Historia16-temas de hoy, núm. 27, 1996, $146 \mathrm{pp}$.

Álvarez Junco, J.: El Emperador del Paralelo. Lerroux y la demagogia populista, Madrid, Alianza Editorial, 1998, $509 \mathrm{pp}$.

Avilés, J.: La izquierda burguesa en la Segunda República. Prólogo de Javier Tusell. Madrid, Espasa Calpe, 1985, 397 pp.

Capitan A.: Historia de la educación en España, Madrid, Dykinson (Col. Pedagogía Contemporánea), 1994, vol. II, 100-1 pp.

CAUDET, F.: Hipótesis sobre el exilio republicano de 1939. Prólogo de Alicia Alted. Madrid, FUE (Col. Archivo 11 de la República española en el exilio), 1997, $541 \mathrm{pp}$.

Cuevas, T.: Cárcel de Mujeres Barcelona, Sirocco Books, 1985, vol. 2, 320 pp.

DoÑA, J.: Desde la noche y la niebla (mujeres en las cárceles franquistas), Madrid, Ediciones La Torre, 1978, $276 \mathrm{pp}$.

Garrido, E. (coord): Historia de las mujeres en España, Madrid, Sintesis, 1997, 607 pp.

Gil Pecharroman, J.: La Segunda República. Esperanzas y fustraciones, Madrid, Historia 16 temas de hoy, núm. 26, 1996, 146 pp.

GonzAlez LópEZ, E.: El espiritu universitario, Madrid, Ediciones Morata, 1931, 113 pp.

Julia, S.: Manuel Azaña, una biografia politica: del Ateneo al Palacio Nacional, Madrid, Alianza Editorial, 1990, 506 pp: (coord), Victimas de la Guerra Civil, Madrid, Temas de Hoy,1999, $431 \mathrm{pp}$

LEON, M. ${ }^{\text {. }}$ T.: La Historia tiene la palabra: noticia sobre el salvamento del Tesoro Artístico, Madrid, Hisparnerca, 1977,122 pp.

LOPEz REY, J.: Los estudiantes frente a la Dictadura, Madrid, Javier Morata editor, 1930, $316 \mathrm{pp}$.

NASH, M. Mujer, Familia y Trabajo en España (1875-1936), Barcelona, Anthropos, (Col. Historia, Ideas y Textos), 1983, $394 \mathrm{pp}$.

Nuñez Pérez, M. a G.: Trabajadoras en la Segunda República. Un estudio sobre la actividad económica extradoméstica (1931-1936), Madrid, M. ${ }^{\circ}$ de Trabajo y Seguridad Social (Col. Tesis Doctorales), 1989, $708 \mathrm{pp}$.

Pamies, T., Los que se fueron, Madrid, Ediciones Martínez Roca, 1976; $197 \mathrm{pp}$.

PAYNe S., El primer franquismo (1939-1956). Los años de autarquia, Madrid, Historia 16-temas de hoy, núm. 28, 1997, 146 pp.

Samaniego, M.: La política educativa de la Segunda República durante el bienio azañista. Prólogo de M.a Dolores Gómez Molleda. Madrid, CSIC, 1977, xxxI, 392 pp.

SánCHEZ RoN, J.M. (coord): La Junta para la Ampliación de Estudios e Investigaciones Cientificas 80 años después, 1907-1987, Simposio Internacional (Madrid, 15-17 de diciembre, 1987), Madrid, CSIC, 1988, 2 vol. 
SORIA, A.: “ Ortega: primer y único encuentro", Revista de Occidente, núm. 26 junio julio 1983), pp. 79-86.

Townson, N. ( eds.): El republicanismo en España (1830-1977), Madrid, Alianza Universidad, $1994,453 \mathrm{pp}$

Tusell, J; Alted, A y MAteos, A (coords): La oposición al régimen de Franco. Estado de la cuestión y metodologia de la investigación, Madrid, UNED, Departamento de Historia Contemporánea 1990, Tomo II, $688 \mathrm{pp}$.

VVAA: Los origenes culturales de la /l República. Coloquio de Historia Contemporánea de España. Dirigido por Manuel Tuñon de Lara. (Ix. Cuenca. 1992), Madrid, Siglo Xx।, 1993, $369 \mathrm{pp}$. 